\title{
Development of a nanoporous and multilayer drug-delivery platform for medical implants
}

\author{
This article was published in the following Dove Press journal: \\ International Journal of Nanomedicine \\ 5 October 2012 \\ Number of times this article has been viewed
}

\section{Varvara Karagkiozaki' \\ Eleftherios Vavoulidis' \\ Panagiotis G Karagiannidis \\ Maria Gioti' \\ Dimitrios G Fatouros ${ }^{2}$ \\ loannis S Vizirianakis ${ }^{3}$ \\ Stergios Logothetidis'}

'Lab for Thin Films-Nanosystems and Nanometrology, Physics Department,

${ }^{2}$ Department of Pharmaceutical Technology, ${ }^{3}$ Laboratory of

Pharmacology, School of Pharmacy, Aristotle University of Thessaloniki, Greece
Correspondence: Stergios Logothetidis Aristotle University of Thessaloniki, Physics Department, Laboratory for Thin Films-Nanosystems and Nanometrology, GR-54I24, Greece

Tel +302310998174

Fax +302310998390

Email logot@auth.gr
Abstract: Biodegradable polymers can be applied to a variety of implants for controlled and local drug delivery. The aim of this study is to develop a biodegradable and nanoporous polymeric platform for a wide spectrum of drug-eluting implants with special focus on stent-coating applications. It was synthesized by poly(DL-lactide-co-glycolide) (PLGA 65:35, PLGA 75:25) and polycaprolactone (PCL) in a multilayer configuration by means of a spin-coating technique. The antiplatelet drug dipyridamole was loaded into the surface nanopores of the platform. Surface characterization was made by atomic force microscopy (AFM) and spectroscopic ellipsometry (SE). Platelet adhesion and drug-release kinetic studies were then carried out. The study revealed that the multilayer films are highly nanoporous, whereas the single layers of PLGA are atomically smooth and spherulites are formed in PCL. Their nanoporosity (pore diameter, depth, density, surface roughness) can be tailored by tuning the growth parameters (eg, spinning speed, polymer concentration), essential for drug-delivery performance. The origin of pore formation may be attributed to the phase separation of polymer blends via the spinodal decomposition mechanism. SE studies revealed the structural characteristics, film thickness, and optical properties even of the single layers in the triple-layer construct, providing substantial information for drug loading and complement AFM findings. Platelet adhesion studies showed that the dipyridamole-loaded coatings inhibit platelet aggregation that is a prerequisite for clotting. Finally, the films exhibited sustained release profiles of dipyridamole over 70 days. These results indicate that the current multilayer phase therapeutic approach constitutes an effective drug-delivery platform for drugeluting implants and especially for cardiovascular stent applications.

Keywords: drug delivery, implants, stents, polymers, spin-coating, atomic force microscopy

One of the fields that has benefited from nanomedicine is the design of new drug-delivery and -release systems. These novel systems can be combined with state-of-the-art implant technology and, in turn, give birth to drug-eluting implants that release therapeutic agents at the site of implantation. One of the most promising categories of drug-eluting coatings is considered to be nanoporous platforms with pore sizes less than $0.1 \mu \mathrm{m}$. Porosity of such low dimensions contributes to the material's high active surface and drug loading, leading to desirable drug release profiles for each medical application. ${ }^{1,2}$

A wide range of different materials and fabrication methods have been used to manufacture nanoporous coatings. ${ }^{3}$ Biocompatible metals and their alloys, such as titanium and aluminum, are subjected to a self-ordering porous formation that is based on electrochemical processes. ${ }^{4}$ Polymers and especially block copolymers have been widely used to compose nanoporous drug-delivery platforms aimed at 
clinical applications. ${ }^{5,6}$ However, their use has been hindered because of limited biodegradability and slow degradation rates. ${ }^{7}$ These properties were found to cause undesirable side effects, such as hypersensitivity reactions at the site of implantation, inflammation, and thrombus formation, which can lead to tissue damage and even implant failure. ${ }^{8}$

In this study, we designed and developed biodegradable polymeric matrices in a multilayer configuration characterized by a diversity of nanopores for controlled drug loading and release. Two classes of poly(DL-lactide-co-glycolide) (PLGA 65:35, PLGA 75:25) and polycaprolactone (PCL) with different degradation rates constitute the nanolayers of the platform, and they were deposited by spin coating. Although this technique has been widely used for the deposition of thin films for various applications, ${ }^{9,10}$ to the best of our knowledge this is the first time that it has been used for the synthesis of drug-eluting applications. In particular, novel nanoporous materials with a variety of nanopore characteristics (depth, density, and diameter) were manufactured to serve as drug reservoirs with multiplex loading capacities. The major goals in designing drug-delivery systems are to control nanopore size, surface properties, and release of pharmacologically active agents in order to achieve the site-specific action of the drug at the nanoporosity therapeutically optimal rate and dose regimen. The control of the nanoporosity of the engineered nanomaterials was achieved by the implementation of highly sensitive techniques, such as atomic force microscopy (AFM) and spectroscopic ellipsometry (SE) in correlation with variations in deposition parameters.

These multilayer polymeric nanocoatings may serve as a drug-eluting platform for a wide spectrum of implants (eg, orthopedic, cardiovascular, retinal etc). The design of the platform and the selection of polymers and drugs should be made in line with the specific medical application. In this study, the platform was designed for stent-coating needs.

Several drug candidates, such as immune-suppressive agents, anti-inflammatory, and cellular proliferation inhibitors, have been employed for stent coating and evaluated in clinical trials. Sirolimus-eluting $\left(\right.$ Cypher $^{\circledR}$; Cordis, Miami Lakes, FL) and paclitaxel-eluting (Taxus ${ }^{\circledR}$; Boston Scientific, Natick, MA) stents have been extensively used for coronary angioplasty. ${ }^{11,12}$

In our study, dipyridamole (DPM), an antiplatelet drug known to inhibit clotting, ${ }^{13}$ was encapsulated into the external layer of the polymeric matrix and the release of the drug was monitored over time. We used platelet adhesion studies to assess their antithrombogenic effect.

\section{Methods}

\section{Materials}

Dipyridamole was obtained from Sigma-Aldrich (Athens, Greece). $\mathrm{NaCl}, \mathrm{KCl}, \mathrm{KH}_{2} \mathrm{PO}_{4}$, and $\mathrm{Na}_{2} \mathrm{HPO}_{4}$ were obtained from Merck (Darmstadt, Germany). PLGA with different lactide:glycolide contents (65:35 with average molecular weight $\left[\mathrm{M}_{\mathrm{w}}\right]=40,000-75,000$ and $75: 25$ with average $\mathrm{M}_{\mathrm{w}}=66,000-107,000$ ) and PCL (with average $\mathrm{M}_{\mathrm{w}}=48,000-$ 90,000) were purchased from Sigma-Aldrich. All solutions were prepared with deionized water.

\section{Fabrication of multilayer films}

The samples were fabricated by spin coating onto silicon (Si) and stainless steel substrates inside a nitrogen-filled glovebox. For the fabrication of polymeric single layers, a solution of the corresponding polymer was prepared with a total concentration of $10 \mathrm{mg} \mathrm{mL}^{-1}$ in chloroform. The solution was spin coated under various experimental conditions (rotation speed $=3-95 \times g$ and spinning time $=18-30$ seconds $)$. The substrates were cleaned prior to spin coating with isopropanol and methanol and blow dried using $\mathrm{N}_{2}$ flow. Each layer was left overnight to slow dry any residual solvent left before the deposition of the next polymeric layer.

\section{Drug-loading studies}

A solution of drug (PLGA [65:35] 1:3, weight [w]/w) was prepared with a total concentration of $13.3 \mathrm{mg} \mathrm{mL}^{-1}$ in chloroform. The initially added amount of the drug was $1.0 \mathrm{mg}$. The amount of the drug remaining in the film after the spin-coating method was determined by washing the substrate with $3 \mathrm{~mL}$ of $\mathrm{CHCl}_{3}$ and measuring the free drug with a UV-1700 ultraviolet (UV)-visible spectrophotometer (Shimadzu, Kyoto, Japan) at $292 \mathrm{~nm}$.

\section{Atomic force microscopy for platelet adhesion studies}

The polymeric layers were imaged by an AFM Solver P-47H (NT-MDT, Moscow, Russia) scanning probe microscope at ambient environmental conditions. For platelet adhesion studies, human platelet-rich plasma (PRP) was prepared after the centrifugation of whole blood drawn by venopuncture (kept in tubes with $3.8 \%$ citrate acid) from healthy donors at $4 \times \mathrm{g}$ for 7-10 minutes at room temperature. The polymeric films were cleaned by $\mathrm{N}_{2}$ flow and incubated in PRP at room temperature. AFM was then applied for platelet visualization onto the drug-free triple layers (PLGA [65:35]-PLGA [75:25]-PCL) and DPM-loaded ones (PLGA [65:35] + DPM-PLGA [75:25]-PCL) at 1- and 
2-hour intervals in the tapping mode for better image acquisition and avoidance of platelet damage. ${ }^{14}$ The quantities that were used for the evaluation of surface roughness of the multilayer films before and during platelet adhesion were peak-to-valley $\left(R_{y}\right)$ distance and root-mean-square roughness $\left(\mathrm{R}_{\mathrm{q}}\right)$. Ten areas of the samples were chosen at random to obtain statistical averages of the $R_{q}$ and $R_{y}$ parameters by Student's $t$-test.

\section{Spectroscopic ellipsometry studies}

The optical properties of the PLGA and PCL films were studied with the SE technique using a phase-modulated spectroscopic ellipsometer (by Horiba/Jobin-Yvon), covering the extended spectral range from near-infrared to far UV (1.5-6.5 eV). It has already been established that $\mathrm{SE}$ is a suitable technique for measuring the optical constants of the materials..$^{15}$ However, few data have been reported on the optical characterization of polymeric thin films using SE. Ellipsometry measures changes in the reflectance and phase difference between the parallel $\left(R_{p}\right)$ and perpendicular $\left(\mathrm{R}_{\mathrm{s}}\right)$ components of a polarized light beam upon reflection from a material surface. Using the following equation:

$$
\tan (\Psi) e^{i \Delta}=\frac{\mathrm{R}_{\mathrm{p}}}{\mathrm{R}_{\mathrm{s}}}
$$

The intensity ratio of $\mathrm{R}_{\mathrm{p}}$ and $\mathrm{R}_{\mathrm{s}}$ can be related to the amplitude ratio $(\Psi)$ and the phase difference $(\Delta)$ between the two components of polarized light. ${ }^{15}$ Because ellipsometry measures the ratio of two values originating from the same signal, the data collected are accurate and reproducible. Moreover, the changes in polarization measured by ellipsometry are extremely sensitive to thickness (down to monolayer level), microstructure, and optical properties of the film under study.

\section{In vitro drug release studies}

The multilayer polymeric films $(n=7$ indicates the number of substrates coated with the polymeric films) loaded with dipyridamole were immersed in phosphate buffered saline (PBS) at $37^{\circ} \mathrm{C}$ for 70 days in order to determine the drugrelease kinetics. The release studies were conducted in 24-well cell culture clusters (Costar; Corning, Manassas, VA) containing $1.0 \mathrm{~mL}$ of PBS ( $\mathrm{pH} 7.4)$. Sodium azide $(0.05 \%$ $\mathrm{w} /$ volume) was added to the medium in order to prevent bacterial growth. The medium was removed (completely) at each sampling time $(2,6$, and 12 hours and 1, 2, 3, 5, 7, 14,
21, 28, 35, 49, 56, and 70 days) assayed with UV at $292 \mathrm{~nm}$ and fresh medium introduced.

\section{Results}

\section{Surface nanotopography and structural properties of single- and multilayer- polymeric films}

The surface morphology of the polymeric films was investigated by AFM. A series of single-layer polymeric films was first fabricated. Figure 1A illustrates the topography image of a single-layer film made of PLGA (75:25), while the topography image of a single-layer film made of PCL is depicted in Figure 1B. The AFM images revealed that the PLGA films were atomically smooth with a surface roughness of $0.2 \mathrm{~nm}$ under variable experimental conditions. In contrast to the PLGA films, the PCL films (Figure 1B) were less smooth with a surface roughness of ca. $5.5 \mathrm{~nm}$. The surface topography demonstrated the formation of spherulites with various sizes $(5-40 \mathrm{~nm})$ as an outcome of the crystallization process that takes places after thin-film deposition. In Figure 1E, the corresponding $X$ cross sections of Figure 1B and $\mathrm{C}$ indicate that the holes between the spherulites of the PCL single layers reaches ca. $8 \mathrm{~nm}$ and the pore depth in the dual layers is ca. $10 \mathrm{~nm}$.

After fabricating single-layer samples, dual-polymeric layers made of PLGA (as the outer layer) and PCL (as the inner layer) were constructed. Figure $1 \mathrm{C}$ presents the topography image of a dual-layer polymeric film. In the dual-layer polymeric films, the formation of nanopores was evident. Characterization of the pores revealed variation in diameter (20-170 nm) and in depth (2-17 $\mathrm{nm})$. The pore density was estimated between 40 and 70 pores $/ \mu \mathrm{m}^{2}$ under different experimental conditions. Triple-layer polymeric films composed of PLGA (65:35; outer layer), PLGA (75:25; intermediate layer), and PCL (inner layer) were then manufactured. Figure 1D presents the topography image of a triple-layer film. Nanopores were observed in the triple layers as well. Hence, the AFM data demonstrated the formation of nanopores with smaller diameter $(20-150 \mathrm{~nm})$ and depth compared to the pores formed onto the surface of dual-layer polymeric matrices. The pore density of these triple layers was estimated between 20 and 80 pores $/ \mu \mathrm{m}^{2}$. The AFM data involving the surface roughness parameters and pore characteristics of all layers are presented at Table 1.

In an attempt to investigate to what extent the process of pore formation can be controlled, the triple-layer 

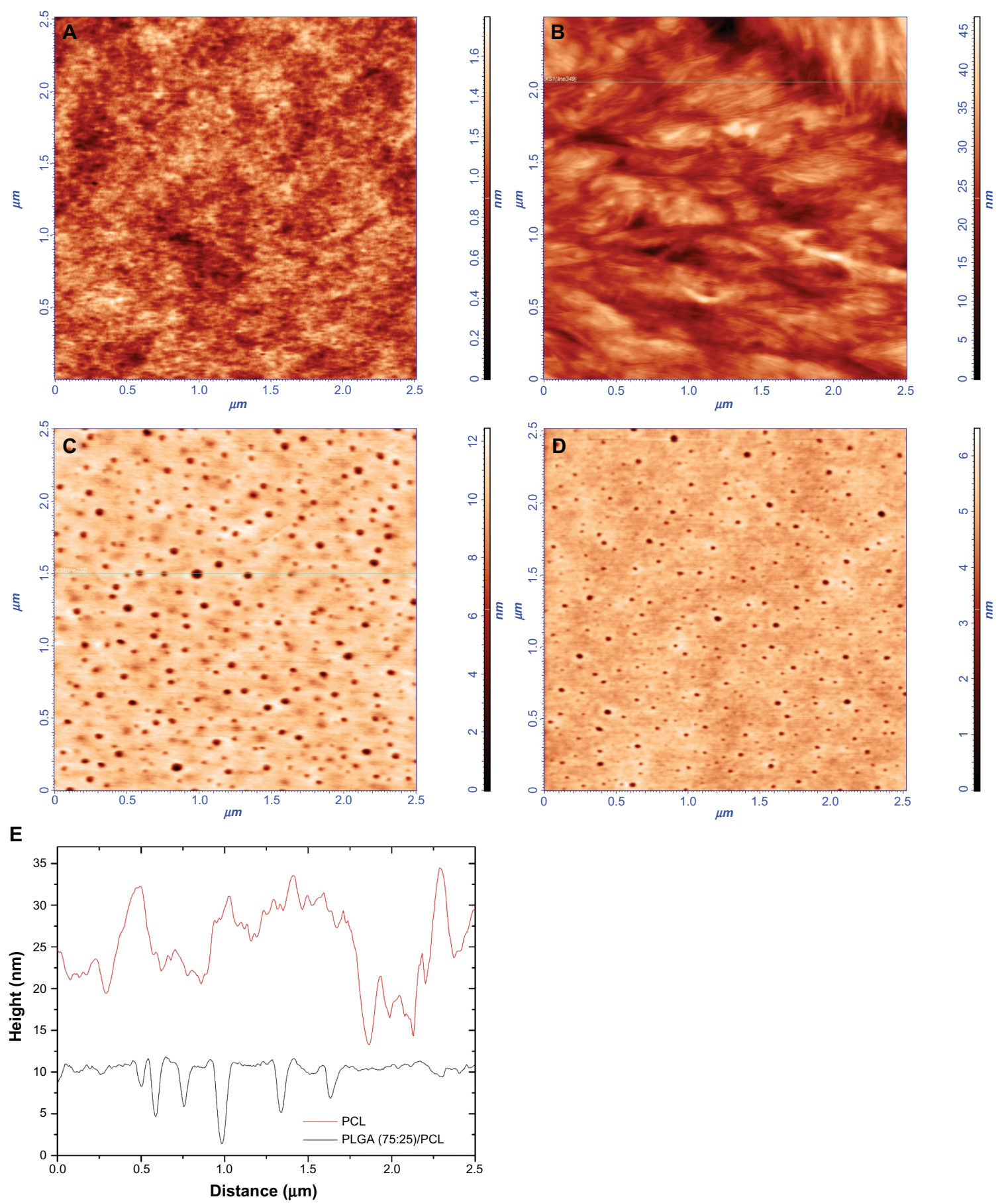

Figure I AFM topography images of: (A) PLGA single layer that was spin coated at $53 \times g$ for 30 seconds. (B) PCL single layer that was spin coated at $53 \times g$ for 30 seconds. (C) Dual layer (PLGA [75:25]-PCL) that was spin coated at $53 \times \mathrm{g}$ for 30 seconds. (D) Triple layer (PLGA [65:35]-PLGA [75:25]-PCL) that was spin coated at 53 $\times \mathrm{g}$ for 30 seconds. The scan size is $2.5 \mu \mathrm{m} \times 2.5 \mu \mathrm{m}$. (E) Corresponding $X$ cross sections of Figure IB and $\mathrm{C}$ that reveal the dimensions of the holes between the spherulites of $\mathrm{PCL}$ single layers and the pore sizes of PLGA-PCL dual layers, respectively.

Abbreviations: PLGA, poly (DL-lactide-co-glycolide); PCL, polycaprolactone.

films were fabricated under variable experimental conditions: (a) By altering the spinning speed at $24 \times \mathrm{g}$ and $53 \times \mathrm{g}$, two series of triple-layer films were developed. Figure 2 depicts the AFM topography images of triple-layer films, spin coated at $24 \times \mathrm{g}$ for 30 seconds and at $53 \times \mathrm{g}$ for 30 seconds. Both samples had a polymer concentration of $10 \mathrm{mg} \mathrm{mL}^{-1}$; (b) by variations in polymer concentration of the outer layer $(5 \mathrm{mg}$ $\mathrm{mL}^{-1}$ and $10 \mathrm{mg} \mathrm{mL}^{-1}$ ). Figure 2 shows the topography images of triple-layer films, one with a polymer concentration of $10 \mathrm{mg} \mathrm{mL}^{-1}$ and the other with a concentration of $5 \mathrm{mg} \mathrm{mL} \mathrm{m}^{-1}$. Both samples were spin coated at $24 \times \mathrm{g}$ for 30 seconds. These two parameters were correlated with the 
Table I AFM data of the surface characteristics of the drug-free polymeric layers

\begin{tabular}{llclll}
\hline & $\mathbf{R}_{\mathbf{q}}(\mathbf{n m})$ & $\mathbf{R}_{\mathbf{y}}(\mathbf{n m})$ & $\begin{array}{l}\text { Pore depth } \\
\mathbf{( n m )}\end{array}$ & $\begin{array}{l}\text { Pore diameter } \\
(\mathbf{n m})\end{array}$ & $\begin{array}{l}\text { Pore density } \\
\left(\mathbf{p o r e s} \mu \mathbf{m}^{-2}\right)\end{array}$ \\
\hline Single layers & & & & - & - \\
PLGA 75:25 & $0.2 \pm 0.02$ & $2.1 \pm 0.3$ & - & - & - \\
PCL & $5.5 \pm 1$ & $55 \pm 16$ & - & $20-170$ & $40-70$ \\
Dual layers & $1.1 \pm 0.3$ & $14.2 \pm 3$ & $2-17$ & $20-150$ & $20-80$ \\
Triple layers & $0.3 \pm 0.06$ & $7 \pm 3$ & $1-7$ & \\
\hline
\end{tabular}

Abbreviations: AFM, atomic force microscopy; PLGA, poly (DL-lactide-co-glycolide); PCL, polycaprolactone; $R$, peak-to-valley distance; $R$, root mean square roughness.

surface roughness, pore depth, diameter, and pore density of the engineered triple layers (Table 2).

\section{Spectroscopic ellipsometry studies for optical characterization of polymeric layers}

SE was successfully employed for determining the optical properties, thickness, and structural characteristics of the spin-coated polymeric films, either in the form of single-, dual-, or triple-layer structure. The optical model that was applied to analyze the measured data consisted of the appropriate number of layers for each case. For determining the optical properties and the thickness of the films, a standard fitting procedure was applied in which the dielectric function $\varepsilon(\omega)$ of the polymeric films was described using the Tauc-Lorentz (TL) oscillator dispersion equation, where the imaginary part of the dielectric function is given by

$$
\varepsilon_{2}(\omega)=\frac{\mathrm{A} \omega_{0} \mathrm{C}\left(\omega-\omega_{\mathrm{g}}\right)^{2}}{\left(\omega^{2}-\omega_{0}^{2}\right)^{2}+\mathrm{C}^{2} \omega^{2}} \cdot \frac{1}{\omega}, \omega>\omega_{\mathrm{g}}
$$

where $\omega$ is the photon energy, $\omega_{\mathrm{g}}$ the fundamental band gap energy, A the amplitude of the oscillator, $\omega_{0}$ the Lorentz resonant energy, $\mathrm{C}$ its broadening term, and the real part $\varepsilon_{1}(\omega)$ is obtained by the Kramer-Kronig integration. ${ }^{16}$

The real part of the refractive index (n) and the extinction coefficient $(\mathrm{k})$ of the complex refractive index are related to the dielectric function by the following equations: ${ }^{16}$

$$
\varepsilon_{1}=\mathrm{n}^{2}-\mathrm{k}^{2}
$$

and

$$
\varepsilon_{2}=2 \mathrm{nk}
$$

Figure 3 shows the experimentally measured pseudodielectric function $\langle\varepsilon(\omega)>$ (curves with symbols) of single PLGA 75:25, PCL, dual-(PLGA [75:25]-PCL) 75:25-PCL films, and triple-(PLGA [65:35]-PLGA [75:25]-PCL) films that were spin coated at $53 \times \mathrm{g}$. The $\langle\varepsilon(\omega)>$ accounts for the effect of film thickness and the substrate in addition to the dielectric response of the bulk film, and this is evident by the multiple reflections that appear in the spectra. The multiplex reflections are expected to be eliminated when the penetration depth of light becomes smaller than the film thickness. However, in our cases the whole spectra are dominated by multiple reflections since the PLGA and PCL
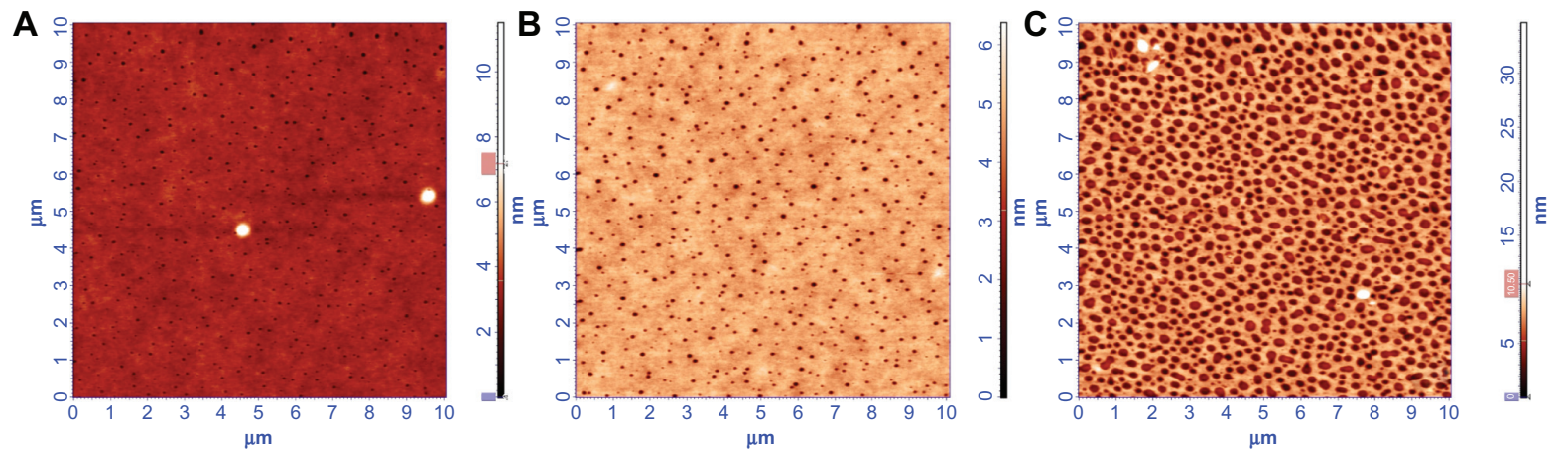

Figure 2 AFM topography images of the triple layer (PLGA [65:35]-PLGA [75:25]-PCL) fabricated under variable experimental conditions. (A) Polymer concentration of $10 \mathrm{mg} \mathrm{mL} \mathbf{L}^{-1}$, spin coated at $24 \times \mathrm{g}$ for 30 seconds; (B) polymer concentration of $10 \mathrm{mg} \mathrm{mL}^{-1}$, spin coated at $53 \times \mathrm{g}$ for 30 seconds; and (C) polymer concentration of $5 \mathrm{mg} \mathrm{mL}^{-1}$, spin coated at $24 \times \mathrm{g}$ for 30 seconds.

Note: The scan size is $10 \mu \mathrm{m} \times 10 \mu \mathrm{m}$.

Abbreviations: PLGA, poly (DL-lactide-co-glycolide); PCL, polycaprolactone. 
Table 2 AFM data of triple layers (PLGA [65:35]-PLGA [75:25]-PCL) fabricated under variable experimental conditions

\begin{tabular}{|c|c|c|c|c|c|}
\hline $\begin{array}{l}\text { Polymer concentration } \\
\left(\mathrm{mg} \mathrm{mL}^{-1}\right)\end{array}$ & $\begin{array}{l}\text { Spinning speed } \\
\text { (rpm) }\end{array}$ & $\begin{array}{l}\text { Pore depth } \\
(\mathrm{nm})\end{array}$ & $\begin{array}{l}\text { Pore diameter } \\
(\mathrm{nm})\end{array}$ & $\begin{array}{l}\text { Pore density } \\
\left(\text { pores per } \mu \mathrm{m}^{2}\right)\end{array}$ & $R_{q}(n m)$ \\
\hline 10 & $24 \times g$ & $1-7$ & $20-120$ & 25 & 0.4 \\
\hline 10 & $53 \times g$ & $1-6$ & $20-150$ & 20 & 0.5 \\
\hline 5 & $24 \times g$ & $3-8$ & $20-310$ & 43 & 1.8 \\
\hline
\end{tabular}

Abbreviations: AFM, atomic force microscopy; PLGA, poly (DL-lactide-co-glycolide); PCL, polycaprolactone; $R_{q}$, root mean square roughness.

start to absorb at energies above the upper experimental energy limit. In Figure 3, solid lines at the respective plots correspond to the simulated spectra derived by the applied fitting procedure. By this procedure, the films' thicknesses, the bulk dielectric functions, and the refractive indices of the produced PLGA and PCL films were calculated. In Figure 4 the corresponding real part of the complex refractive index $n$, referred to the bulk materials, has been plotted; we omitted the contribution of the thickness and the substrate, and the best-fit parameters of the TL oscillator model were used to reproduce the dielectric function or refractive index of the PLGA and PCL materials.

The AFM data obtained for the single-layer films were in agreement with the SE data analysis since for the case of PLGA films there was no need to introduce an additional layer in the applied geometrical model (air/film/substrate) for the analysis in order to take into account the surface roughness. Indeed, the PLGA films were found to be atomically smooth.

In contrast, better fitting analysis was established by the introduction of a four-phase model (air/surface layer/
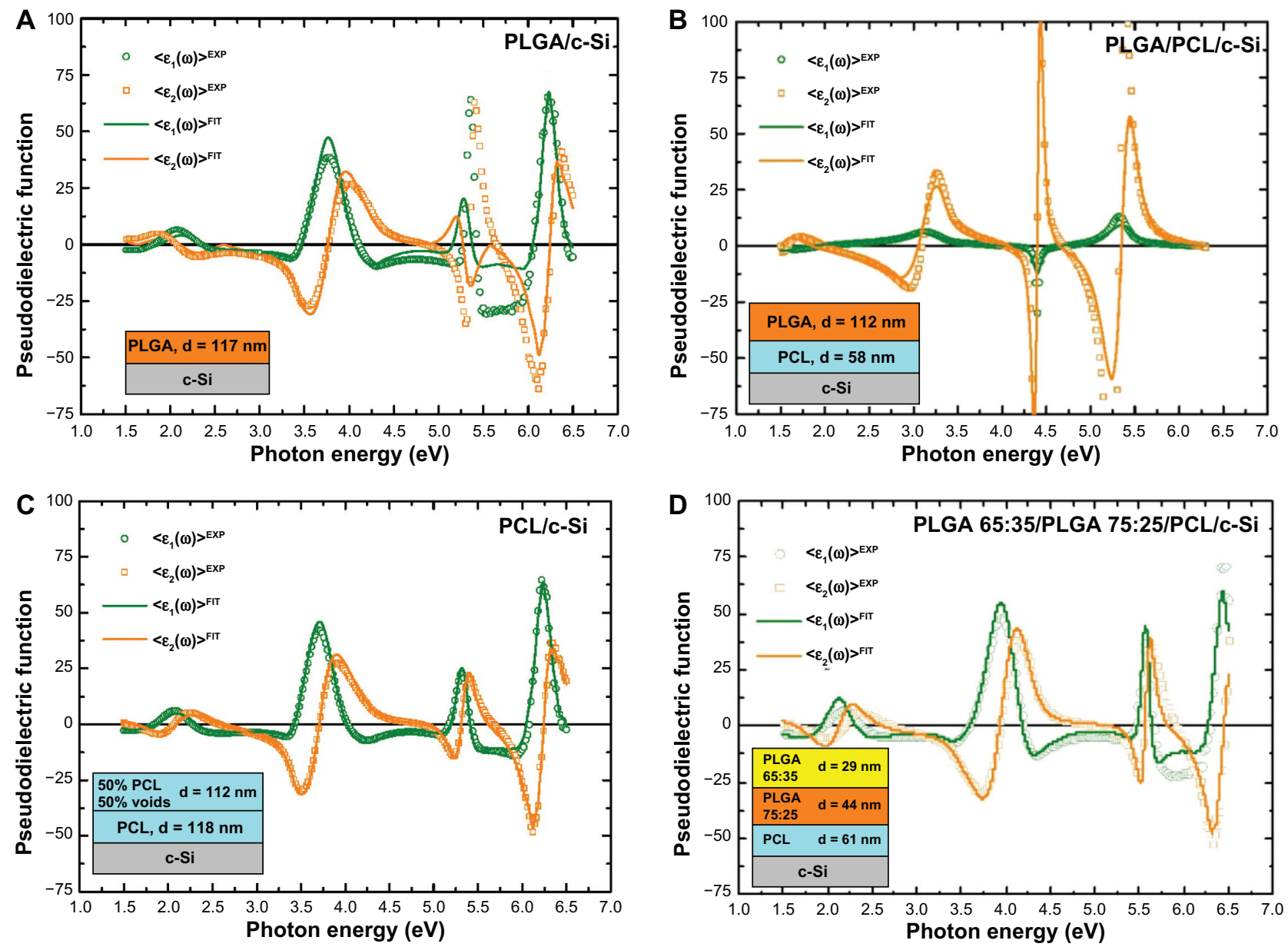

Figure 3 The experimental pseudodielectric functions (symbols) and the corresponding simulated ones (solid lines) determined by the use of best-fit results or the single-layer polymeric films: (A) PLGA 75:25-c-Si, (B) PCL-c-Si, (C) the dual-layer PLGA (PLGA [75:25]-PCL-c-Si), and (D) the triple-layer (PLGA [65:35]-PLGA [75:25]-PCL-c-Si).

Note: The insets in the figures show the geometrical model, which was applied in each case, and the respective thicknesses of the polymeric films derived by the fitting analysis.

Abbreviations: PLGA, poly (DL-lactide-co-glycolide); PCL, polycaprolactone; Si, silicon. 


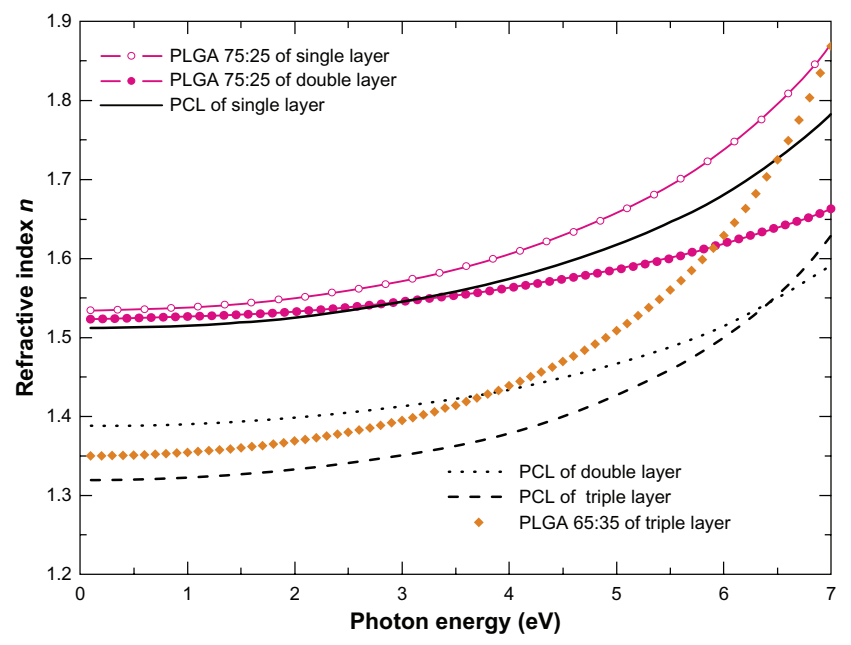

Figure 4 The real part of the bulk complex refractive index $n$ calculated using bestfit parameters of the SE data analysis for the single-layer polymeric films PLGA 75:25 and PCL, the PLGA 75:25 and PCL from the dual-layer film, and the PLGA 65:35 and $\mathrm{PCL}$ from the triple-layer film.

Abbreviations: SE, spectroscopic ellipsometry; PLGA, poly (DL-lactide-co-glycolide); $\mathrm{PCL}$, polycaprolactone.

film/substrate) for the case of PCL films (see also inset in Figure $3 \mathrm{~B}$ ). With this model analysis, the surface roughness was represented by a top layer that is composed of polymeric material (PCL) and air (voids) with an equal percentage of $50 \%$. The calculated effective thicknesses of these rough layers for the variable PCL films varied from 2.5 to $8.6 \mathrm{~nm}$.

The formation of nanopores or voids in the dual- (PLGA 75:25-PCL) and triple-layer films (PLGA 65:25-PLGA 75:25-PCL) was also verified by the SE studies. More specifically, from Figure 4 where the bulk refractive index $n$ has been plotted versus photon energy, nanopores formation is evident from the reduction of the $n$ values in both PLGA and PCL films that consist of dual- and triple-layer polymeric film compared to those derived for the single-layer films. The decrease in $n$ values may be due to the reduction in the density of the material and partially due to the surface and interface roughness in PCL and PCL-PLGA layers. Moreover, it is noticeable that a more drastic reduction in the $n$ values was deduced for the PCL films compared to the PLGA. Therefore, we can interpret the SE results with nanopore formation in the dual- and triple-layer films.

\section{Platelet adhesion studies onto drug-free and drug-loaded triple-polymeric layers}

In order to perform platelet adhesion studies, drug-free and dipyridamole-loaded triple-layer films (PLGA 65:35-PLGA 75:25-PCL) were synthesized (Figures $1 \mathrm{D}$ and 5A, respectively). The DPM-loaded triple layer was characterized by a slight increase of surface roughness $\left(R_{y} 12 \mathrm{~nm}\right.$ and $R_{q}$ $1.4 \mathrm{~nm})$ compared to the drug-free one $\left(\mathrm{R}_{\mathrm{y}} 9.5 \mathrm{~nm}\right.$ and $\mathrm{R}_{\mathrm{q}}$ $0.3 \mathrm{~nm}$ ) (Table 3). As it is essential to monitor the polymer degradation of this triple layer when it comes in contact with plasma, the DPM-loaded triple layer was placed in PBS solution for 15 minutes (Figure 5B). Interestingly, the PLGA polymer starts to degrade even at this initial stage causing the initiation of drug release and an increase of pores diameter and surface roughness of the material $\left(\mathrm{R}_{\mathrm{y}}: 310 \mathrm{~nm}\right.$ and $R_{q}: 64 n m$ of the sample in plasma versus $R_{y}: 12 n m$ and $\mathrm{R}_{\mathrm{q}}: 1.4 \mathrm{~nm}$ in ambient). The topography images of both drug-free and drug-loaded films after 1 hour and 2 hours of platelet adhesion are shown in Figure 6.

Platelets after their contact with biomaterials are activated and undergo the following morphological changes: (i) their shape gets flatter, (ii) the granules are gathered into the center of the cell, forming the pseudo nucleus (egg-like type), and (iii) broad pseudopodia (filopods) extend, which are essential for platelets spreading onto surfaces and for aggregation. ${ }^{17}$ These structural alterations of platelets during activation can be imaged by AFM in detail, as described in previous studies. ${ }^{18}$ The cells then release biochemical compounds (eg, serotonin, thromboglobulin, platelet factor IV, thromboxane) for further activation and aggregation. ${ }^{17}$

In Figure 6A, the platelets after 1 hour of adhesion onto the drug-free sample were found at different stages of activation: (a) egg-like type platelets, less activated, denoted by circles; (b) more activated platelets with pseudonucleus and pseudopodia, as presented by the blue squares; and (c) platelet aggregation (height ca. $411 \mathrm{~nm}$ ) within the red squares, a stage that precedes clot formation. In Figure 6B, the AFM topography image of platelets after 2 hours of adhesion onto the drug-free sample is depicted. It can be easily noticed that the encircled platelets form aggregations reaching the height of ca. $565 \mathrm{~nm}$. In Figure 6C, the platelets onto the drug-loaded samples after 1 hour of adhesion develop pseudopodia and form clusters with a height of ca. $245 \mathrm{~nm}$, and after 2 hours the height of platelet clusters reaches approximately $245 \mathrm{~nm}$ (as measured by the corresponding cross section, inset of Figure 6D).

The surface roughness $\left(R_{q}\right.$ and $\left.R_{y}\right)$ parameters after 1 hour and 2 hours of platelet adhesion on the drug-free sample and DPM-loaded triple-layer films are listed in Table 3.

\section{Drug release kinetics}

The amount of drug remaining in the substrate after the spinning process was estimated to be $2.82 \% \pm 0.40 \%$ of the initial amount. The cumulative release profiles from the triple-layer 

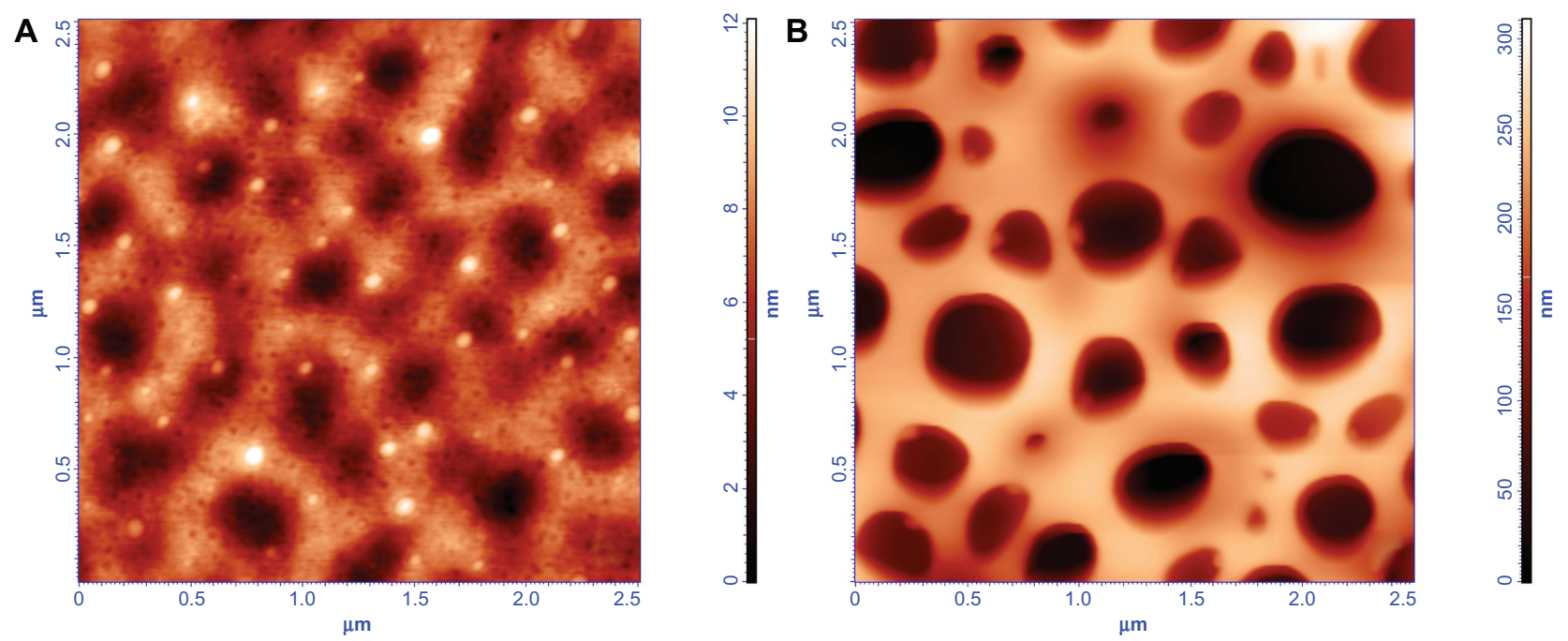

Figure 5 AFM topography images of the triple layer (PLGA [65:35]-PLGA [75:25]-PCL) loaded with dipyridamole: (A) as fabricated by spin coating at $53 \times \mathrm{g}$ for 30 seconds and (B) after its degradation in PBS solution for 15 minutes.

Note: The scan size is $2.5 \mu \mathrm{m} \times 2.5 \mu \mathrm{m}$.

Abbreviations: AFM, atomic force microscopy; PLGA, poly (DL-lactide-co-glycolide); PCL, polycaprolactone; PBS, phosphate buffered saline

sample are depicted in Figure 7. The release profiles can be divided into four main stages: burst release during the first 5 hours (inset to Figure 7); an exponential release rate during the first week; approximately constant release rate during weeks 2-7; and finally an increased release after week 7 to week 10. This indicates that the assembled therapeutic multilayer was slowly disassembled with a release of DPM in a controlled manner, possibly along with a change in the multilayer thickness. It is likely that the degradation of the hydrophobic core (PLGA) induces the formation of particles, and subsequent partitioning of DPM from the hydrophobic core to an aqueous medium could be mainly responsible for the resulting DPM release patterns.

\section{Discussion}

There are clinical needs for the development of biomaterials for implants with diverse functionalities, such as controllable

Table 3 AFM data of platelet adhesion onto drug-free and drugloaded triple layer (PLGA [65:35]-PLGA [75:25]-PCL)

\begin{tabular}{llll}
\hline & $\begin{array}{l}\text { Before } \\
\text { platelet } \\
\text { adhesion }\end{array}$ & $\begin{array}{l}\text { After I hour } \\
\text { platelet } \\
\text { adhesion }\end{array}$ & $\begin{array}{l}\text { After 2 hours } \\
\text { platelet } \\
\text { adhesion }\end{array}$ \\
\hline $\mathrm{R}_{\mathrm{q}}(\mathrm{nm})$ & & & \\
$\quad$ Drug- free & 0.3 & 52.7 & 58.2 \\
$\quad \begin{array}{l}\text { Drug-loaded } \\
(\mathrm{nm})\end{array}$ & 1.4 & 33.7 & 29.7 \\
$\mathrm{R}_{\mathrm{y}}$ Drug- free & 9.5 & 411.3 & 565.5 \\
$\quad$ Drug-loaded & 12.5 & 246.3 & 245.4 \\
\hline
\end{tabular}

Abbreviations: AFM, atomic force microscopy; PLGA, poly (DL-lactide-coglycolide); $P C L$, polycaprolactone; $R_{y}$, peak-to-valley distance; $R_{q}$, root mean square roughness. and multiplex drug release from their surface, in order to serve different goals in the fight against diseases. For example, in cardiovascular stents the antiplatelet drugs for avoidance of thrombosis and the antiproliferative drugs for inhibition of smooth muscle cells proliferation and restenosis should be eluted at different time intervals and in a controllable manner. Furthermore, few studies have shown that the nonbiodegradable polymeric drug reservoirs that comprise the drug-eluting stents (DES) may cause a chronic inflammation and hypersensitivity of the vessel wall. ${ }^{20,21}$

For that reason, in our study biodegradable polymers were selected for manufacturing the drug-delivery platform. Both PLGA and PCL, which are fully biodegradable due to hydrolysis of their ester bonds during their degradation, were used to form the layers of the platform. Targeting their potential application as coatings for DES, the polymers were deposited with a specific order that is in accordance with their degradation rates. In order to achieve a sustained drug elution profile, we designed the construct in a way that the outer polymeric layer will have the fastest degradation rate, while the inner polymeric layer will need much more time to degrade. The PCL was selected to serve the inner layer since its degradation rate is much slower (greater than 24 months) compared to that of PLGA (around 3-6 months). ${ }^{22}$

As regards the two PLGAs with lactide:glycolide ratios of $65: 35$ and 75:25, it has been shown that the time required for degradation of PLGA is related to the monomers' ratio used in production - the higher the content of glycolide units, the lower the time required for degradation. ${ }^{23}$ As a result, the intermediate layer made of PLGA 75:25 requires 

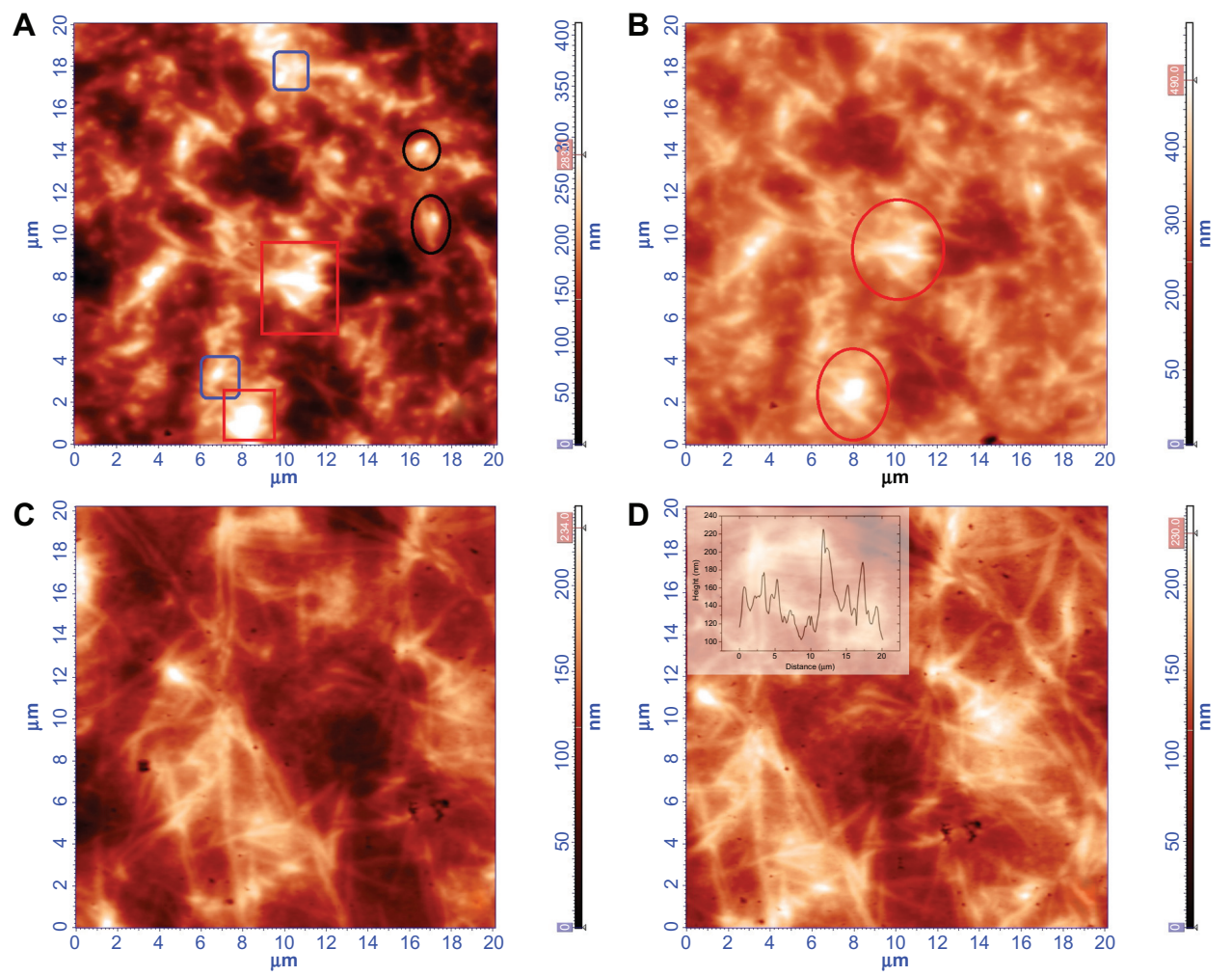

Figure 6 AFM topography images of platelets onto (A and B) drug-free and (C and D) dipyridamole-loaded triple layers (PLGA [65:35]-PLGA [75:25]-PCL). Both samples were spin coated at $53 \times \mathrm{g}$ for 30 seconds. (A) drug-free sample after I hour of platelet adhesion; (B) drug-free sample after 2 hours of platelet adhesion; (C) drug-loaded sample after I hour of platelet adhesion; and (D) drug-loaded sample after 2 hours of platelet adhesion with a typical cross section (inset).

Note: The scan size is $20 \mu \mathrm{m} \times 20 \mu \mathrm{m}$.

Abbreviations: AFM, atomic force microscopy; PLGA, poly (DL-lactide-co-glycolide); PCL, polycaprolactone.

more time to degrade compared to the outer layer made of PLGA 65:35.

In a fundamental drug-release study of biomaterials, an uncontrolled drug release occurred when drug adhered to the outer surface rather than being deposited within the pores. ${ }^{24}$ There are difficulties with control of loading and

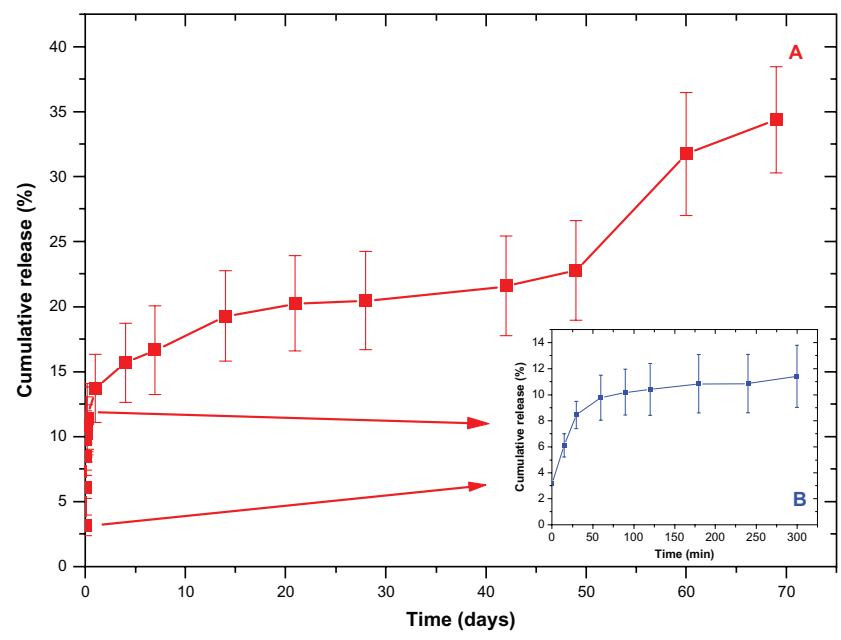

Figure 7 The dipyridamole cumulative release profiles from the three layer samples. Note: The inset graph depicts the burst effect that occurs during the first 5 hours. release of drug from nanometer-sized pores, which is a significant challenge due to their small size. Thus, in this study we describe an experimental procedure for manufacturing multilayer polymeric thin films with tailored nanoporosity for drug loading by the implementation of spin coating. It was noticeable by the data provided by the surface characterization techniques AFM and SE that the PLGA-PCL multilayers were highly nanoporous in contrast to the smooth PLGA and spherulite-characterized PCL single layers.

The origin of pore formation may be attributed to a demixing process, which generally involves the spontaneous phase separation of polymer blend occurring under the specific conditions of spin coating. Similar holes or pores have been reported with a polymer blend phase separating via a spinodal decomposition mechanism. ${ }^{25}$ At the initial stages of spin coating, the upper part of the substrate film was dissolved, forming a blend solution since chloroform is a common solvent for the selected polymers. This hypothesis was verified by the SE data showing that the thickness of the substrate film was reduced after the deposition of the subsequent film (as depicted in Figure 3C and D). 
In addition, Affrossman et $\mathrm{al}^{26}$ reported that morphology evolution is strongly depended on the polymer blend ratio and the film thickness. If the weight content of one component in a polymer blend is much higher (about 90\%) than the other component, then small holes are observed. When this fraction decreases to about $70 \%$, the diameter of the holes increases and the holes start to coalesce. An analogous behavior is observed in our case comparing Figure 2A and $\mathrm{C}$; in Figure 2A the concentration of the added PLGA (65:35) solution is $10 \mathrm{mg} \mathrm{mL}^{-1}$, while in Figure $2 \mathrm{C}$ the concentration is $5 \mathrm{mg} \mathrm{mL}^{-1}$. Since the amount of the added solution is the same in both cases, the reduction in the film thickness and the percentage analogy of the two types of PLGA in the latter case explains its characteristic morphology (Figure 2C).

The pore distribution in the nanoporous biomaterials is a key factor for determination of drug release kinetics and directional control. By varying the size (ie, diameter, volume) and number of the porous reservoirs, a range of therapeutic agent loading levels can be achieved..$^{25}$ In the case of DES where the drug targets different sites of the vessel, it is necessary to control the porosity of the stent nanocoatings for directional and tailored drug loading and resultant release. In this study we found that by tuning the spin-coating parameters, the pore distribution and dimensions, interconnectivity, and density of the materials can be controlled. The AFM images demonstrated that by increasing the spinning speed, the pore diameter and surface roughness were increased, whereas the pore density was decreased. The pore depth was not found to be affected by this parameter, as shown in Table 2. Moreover, the decrease in the polymer concentration of the outer layer of PLGA (65:35), keeping the rotation speed and spinning time constant, resulted in an increase in pore diameter, pore density, and surface roughness of the engineered biomaterials (as presented at Table 3).

In controlled polymeric drug-delivery systems, the drugdelivery rates are mainly determined by the dynamics of polymer degradation, which is strongly related to polymer structure, morphology, and properties. All these can be correlated to the optical properties of the polymeric films. The study of the optical properties and the consequent creation of a database containing specific optical constants of the candidate polymeric films for such systems can provide a powerful tool for a nondestructive and creditable methodology to evaluate their performance. Hence, one can consider that the complete elucidation of the optical response of the engineered systems is essential for the achievement of their functionality as well as for the prediction of their effectiveness.

In this work we show successful implementation of SE for determining the thicknesses, nanoporosity and the optical constants of the spin-coated polymeric films. In regards to thickness measurements, the SE data revealed (Figure 3C and D) that the thickness of the substrate film was reduced after the deposition of the subsequent film due to a spinodal decomposition mechanism.

Dipyridamole was selected for loading within the nanopores of the polymeric multilayers to examine their antiplatelet effect in order to be used as potential coatings for cardiovascular implants. The ultimate goal was to inhibit thrombus formation, which may lead to implant failure and patient complications. The DPM burst effect and release profile during the first week was typical of diffusion-controlled systems. The third and fourth phase of the constant release rate presumably involved degradation of PLGA combined with diffusion of the remaining drug that was more firmly attached to polymer.

As polymer degradation is an important factor that determines drug elution, the AFM enables us to image the degraded DPM-loaded triple-polymeric layer after placing it in PBS solution for 15 minutes. At this initial stage, an increase in surface roughness of the drug-loaded layer was observed due to the degradation of PLGA, the formation of particles occurred, and there was resultant partitioning of the drug from PLGA, causing drug release.

Our previous studies have shown that AFM is a useful tool for imaging platelets in real time, with high precision and without destroying sensitive cells..$^{27,28}$ The AFM images of platelets onto drug-free, triple-layer, polymeric films showed that the platelets are highly activated, forming pseudopodia and aggregates. From the AFM data presented in Table 3, it can be deduced that there was a time-dependent increase of $\mathrm{R}_{\mathrm{q}}$ and $\mathrm{R}_{\mathrm{y}}$ parameters, indicative of platelet aggregation. Our previous studies showed that the process of platelet adhesion onto nanomaterials that are atomically smooth or have low nanoroughness is time-dependent, resulting in an increase in $\mathrm{R}_{\mathrm{y}}$ due to platelet aggregation. ${ }^{28,29}$

In contrast, in the case of the DPM-loaded triple-layer configuration of the biodegradable polymers, after 1 hour of platelet adhesion, although the cells develop pseudopodia and aggregations, the height is lower and remains at the same level after 2 hours. These findings provide evidence that dipyridamole loaded into the outer layer inhibits the platelet tendency to form high clusters, which are a prestage of thrombus. Moreover, although the triple-layer drug-loaded films are rougher 
compared to the drug-free films $\left(\mathrm{R}_{\mathrm{y}} 12.5 \mathrm{~nm}\right.$ versus $9.5 \mathrm{~nm}$ and $\mathrm{R}_{\mathrm{q}} 1.4 \mathrm{~nm}$ versus $0.3 \mathrm{~nm}$, respectively) after 1 hour of platelet adhesion there is a decrease in surface roughness (as depicted by the decrease in $\mathrm{R}_{\mathrm{y}}$ and $\mathrm{R}_{\mathrm{q}}$ values). This flattening of the surface biomaterials due to platelet adhesion is a phenomenon that shows how platelets behave toward nanomaterials with grooves, pores, and other morphologic irregularities.

\section{Conclusion}

In this study, a multilayer and biodegradable polymeric platform with diverse nanopores for drug-eluting implants was successfully developed. The design of the multilayer drug-eluting coatings was based on polymer degradation rates and properties in order to achieve a long-term and controlled drug elution profile; the spin-coating technique was implemented for this purpose. The spontaneous phase separation of polymer blends during spin-coating conditions leads to the creation of nanopores via the spinodal decomposition mechanism. The formation of nanopores onto the biomaterial surface with tailored characteristics (pore diameter, depth, density, surface roughness) was achieved by tuning the growth parameters.

The complementary AFM and SE studies for determination of structural characteristics, film thickness, and optical properties provide essential information for drug-loading capacities. A case study of dipyridamole loading within the nanopores and platelet studies for evaluation of their antiplatelet effect was carried out. It was found that the dipyridamole-loaded coatings inhibit the platelets tendency to form high aggregations.

In-parallel, drug release kinetics studies shown the release profiles of DPM that indicate that the biodegradable multilayer was slowly degraded with releasing DPM in a controlled manner. By fine tuning the dipyridamole release amount in a desired period, it would be possible to prevent thrombosis and recreate physiologically normal arterial conditions after DPM release.

\section{Acknowledgments}

This work was partially supported by NanoArthroxondros project 09SYN-41-1150.

\section{Disclosure}

The authors disclose no conflicts of interest.

\section{References}

1. Kang H, Kim DJ, Park S, Yoo J, Ryu YS. Controlled drug release using nanoporous anodic aluminum oxide on stent. Thin Solid Films. 2007;515:5184-5187.
2. Orosz KE, Gupta S, Hassink M, et al. Delivery of antiangiogenic and antioxidant drugs of ophthalmic interest through a nanoporous inorganic filter. Mol Vis. 2004;10:555-565.

3. Losic D, Velleman L, Kant K, et al. Self-ordering electrochemistry: a simple approach for engineering nanopore and nanotube arrays for emerging applications. Aust J Chem. 2011;64:294-301.

4. Aninwene GE II, Yao C, Webster TJ. Enhanced osteoblast adhesion to drug-coated anodized nanotubular titanium surfaces. Int J Nanomedicine. 2008;3:257-264.

5. Nuxoll EE, Hillmyer MA, Wang R, Leighton C, Siegel RA. Composite block polymer-microfabricated silicon nanoporous membrane. ACS Appl Mater Interfaces. 2009;1:888-893.

6. Bielecka U, Lutsyk P, Janus K, Sworakowski J, Bartkowiak W. Effect of solution aging on morphology and electrical characteristics of regioregular P3HT FETs fabricated by spin-coating and spray coating. Org Electr. 2011;12:1768-1776.

7. Mansour H, Sohn M, Ghananeem A, DeLuca. P. Materials for pharmaceutical dosage forms: molecular pharmaceutics and controlled release drug delivery aspects. Int J Mol Sci. 2010;11:3298-3322.

8. Hara H, Nakamura M, Palmaz JC, Schwartz RS. Role of stent design and coatings on restenosis and thrombosis. Adv Drug Deliv Rev. 2006;58: $377-386$.

9. Karagiannidis P, Kassavetis S, Pitsalidis C, Logothetidis S. Thermal annealing effect on the nanomechanical properties and structure of P3HT:PCBM thin films. Thin Solid Films. 2011;519:4105-4109.

10. Souza F, Lopes K, Nascente P, Leite E. Nanostructured hematite thin films produced by spin-coating deposition solution: Application in water splitting. Solar Energy Mater and Solar Cells. 2009;93:362-368.

11. Acharya G, Park K. Mechanisms of controlled drug release from drugeluting stents. Adv Drug Deliv Rev. 2006;58:387-340.

12. Garg S, Serruys P. Coronary stents: current status. J Am Coll Cardiol. 2010;56:1-42.

13. Mammen EF. An overview of dipyridamole. Thrombosis Res. 1990;57: $1-3$.

14. Müller D, Anderson K. Biomolecular imaging using atomic force microscopy. Trends in Biotech. 2002;20:545-549.

15. Azzam RMA, Boshara N. Ellipsometry and polarized light. In: Azzam RMA, editor. Ellipsometry and Polarized Light. North Holland: Amsterdam; 1977 San Diego.

16. Jellison GE, Modine FA. Parameterization of the optical functions of amorphous materials in the interband region. Appl. Phys. Lett. 1996; 69(3):371-373.

17. Hartwig JH. Platelet structure. In: Michelson AD, editor. Platelets. Academic Press; San Diego 2002:37-45.

18. Karagkiozaki V, Logothetidis S, Kalfagiannis N, et al. AFM probing platelets activation behavior on titanium nitride nanocoatings for biomedical applications. J Nanomedicine. 2009;5:64-72.

19. Bavry A, Kumbhani D, Helton T, Borek P, Mood G, Bhatt D. Late thrombosis of drug-eluting stents: a meta-analysis of randomized clinical trials. Am J Med. 2006;119:1056-1061.

20. Liistro F, Colombo A. Late acute thrombosis after paclitaxel eluting stent implantation. Heart. 2001;86:262-264.

21. Armentano I, Dottori M, Fortunati E, Mattioli S, Kenny JM. Biodegradable polymer matrix nanocomposites for tissue engineering: a review. Polym Degrad Stab. 2010;95:2126-2146.

22. Lu L, Garcia CA, Mikos AG. In vitro degradation of thin poly(DL-lacticco-glycolic acid) films. J Biomed Mater Res. 1999;46:236-244.

23. Gultepe E, Nagesha D, Sridhar S, Amiji M. Nanoporous inorganic membranes or coatings for sustained drug delivery in implantable devices. Adv Drug Deliv Rev. 2010;62:305-315.

24. Norman J, Desal T. Methods for fabrication of nanoscale topography for tissue engineering scaffolds. Ann Biomed Eng. 2006;34(1):89-101.

25. Affrossman S, Henn G, O’Neill S, Pethrick P, Stamm M. Surface topography and composition of deuterated polystyrene-poly (bromostyrene) blends. Macromolecules 1996;29:5010-5016.

26. Hughes G. Nanostructure-mediated drug delivery. J Nanomedicine. $2005 ; 1: 22-30$. 
27. Karagkiozaki V, Logothetidis S, Lousinian S, Giannoglou G. Impact of surface electric properties of carbon-based thin films on platelets activation for nano-medical and nano-sensing applications. Int $J$ Nanomedicine. 2008;3:461-469.

28. Karagkiozaki V, Logothetidis S, Laskarakis A, Giannoglou G, Lousinian S. AFM Study of the thrombogenicity of carbon-based coatings for cardiovascular applications. Mater Sci Eng B. 2008;152:16-21.
29. Karagkiozaki V, Logothetidis S, Kassavetis S, Giannoglou G. Nanomedicine for the reduction of the thrombogenicity of stent coatings. Int J Nanomedicine. 2010;5:239-248.

\section{Publish your work in this journal}

The International Journal of Nanomedicine is an international, peerreviewed journal focusing on the application of nanotechnology in diagnostics, therapeutics, and drug delivery systems throughout the biomedical field. This journal is indexed on PubMed Central, MedLine, CAS, SciSearch $\AA$, Current Contents ${ }^{\circledR} /$ Clinical Medicine,
Journal Citation Reports/Science Edition, EMBase, Scopus and the Elsevier Bibliographic databases. The manuscript management system is completely online and includes a very quick and fair peer-review system, which is all easy to use. Visit http://www.dovepress.com/ testimonials.php to read real quotes from published authors.

Submit your manuscript here: http://www.dovepress.com/international-journal-of-nanomedicine-journal 